\title{
Improved outcomes in coronary artery bypass grafting with beating-heart techniques
}

\author{
Michael Mack, MD \\ Donna Bachand, RN, PhD \\ Tea Acuff, MD \\ James Edgerton, MD \\ Syma Prince, RN \\ Todd Dewey, MD \\ Mitchell Magee, MD
}

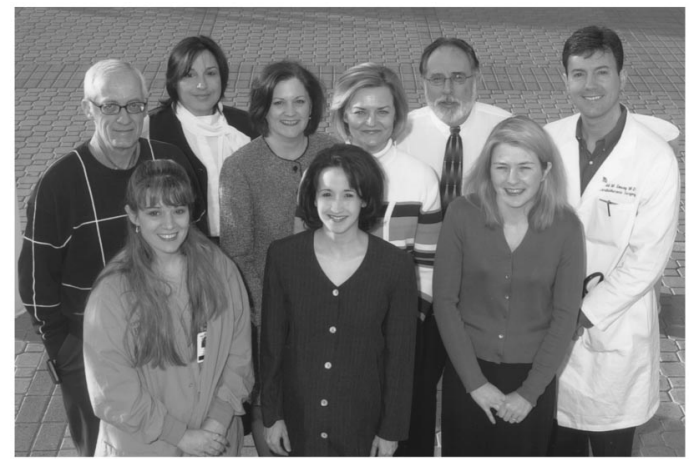

CRSTI Research Staff

Objective: Although improved outcomes for selected patients by elimination of cardiopulmonary bypass have been demonstrated, a benefit for all patients undergoing coronary artery bypass grafting by all surgeons has yet to be definitively proved.

Methods: We reviewed our experience with beating-heart surgery from its inception in January 1995 through December 2000. A total of 12,540 patients underwent isolated coronary artery bypass grafting, including 1915 procedures (15\%) performed without cardiopulmonary bypass. Groups were compared by univariate analysis for preoperative risk factors and postoperative complications, and predicted risk was determined by the Society of Thoracic Surgeons risk algorithm.

Results: There was a gradual increase in the percentage of coronary

See related editorial on page 431.
From the Cardiopulmonary Research Science and Technology Institute (CRSTI), Dallas, Tex.

Read at the Eighty-first Annual Meeting of The American Association for Thoracic Surgery, San Diego, Calif, May 6-9, 2001.

Address for reprints: Michael Mack, MD, 7777 Forest Ln, Suite A323, Dallas, TX 75230 (E-mail: mjmack@earthlink.net).

Received for publication May 15, 2001; revisions requested July 16,2001 ; revisions received Dec 27, 2001; accepted for publication Feb 21, 2002.

J Thorac Cardiovasc Surg 2002;124: 598-607

Copyright $(9) 2002$ by The American Association for Thoracic Surgery

0022-5223/2002 \$35.00+0 $\quad \mathbf{1 2 / 6 / 1 2 4 8 8 4}$

doi: $10.1067 / \mathrm{mtc} .2002 .124884$ operations performed off pump, from $1.2 \%$ in 1995 to $34.1 \%$ in 2000 . Individual surgeon adoption rates ranged from $1 \%$ to $96 \%$ by 2000 . There was a decrease in mortality to $3.22 \%$, compared with an overall observed mortality rate of $4.0 \%$ in the 5 years before beating-heart surgery $(P=.0482)$. There was a significant difference in observed mortality between the off-pump and on-pump groups (1.9\% vs $3.5 \%$, $P<.001)$, despite a higher mean predicted risk among the patients in the off-pump group $(3.13 \%$ vs $2.8 \%, P<.004)$. Additionally, decreased morbidity in the off-pump group was evidenced by reduced needs for blood products $(28.45 \%$ vs $54.65 \%, P=.0001)$, prolonged ventilation $(5.83 \%$ vs $10.93 \%, P=.001)$, and reoperation for bleeding $(2.41 \%$ vs $3.65 \%, P=.0237)$, and by shorter hospital stay (5.98 vs 7.32 days, $P=.001$ ).

Conclusions: Beating-heart surgery can be safely assimilated into a cardiac surgical practice, although adoption rates vary significantly among individual surgeons. Gradual integration can lead to improved outcomes in the total coronary artery bypass surgery population.

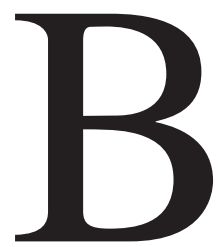

ecause of an increased focus on less invasive coronary artery bypass grafting (CABG) and development of enabling technology and techniques, beating-heart surgery performed without cardiopulmonary bypass (CPB) now comprises more than $20 \%$ of all $\mathrm{CABG}$ procedures performed in North America and Western Europe. ${ }^{1}$ Although only a few, small, randomized studies have demonstrated a benefit from off-pump CABG relative to conventional $\mathrm{CABG}^{2,3}$ a preponderance of retrospective outcomes analysis studies have shown mortality and 
morbidity benefits in specific subgroups undergoing surgical coronary revascularization. Decreased mortality and morbidity, including decreases in postoperative inotropic and intra-aortic balloon pump support, blood loss and transfusion, renal and respiratory failure, and atrial fibrillation, have variously been reported in elderly, ${ }^{4-8}$ high-risk, ${ }^{9,10}$ and reoperative surgical groups. ${ }^{11,12}$ More recently, an analysis of 8499 CABG procedures at the Washington Hospital Center and at our center in Dallas between January 1998 and July 2000, 23\% of which $(n=1983)$ were performed off pump, showed that CABG with CPB had a higher operative mortality than off-pump CABG according to univariate analysis $(3.5 \%$ vs $1.8 \%) .{ }^{13}$

Furthermore, CPB was found to be an independent risk factor for mortality by multiple logistic regression analysis in both unmatched cohorts (odds ratio $1.79,95 \%$ confidence interval [CI] 1.24-2.67) and cohorts computer matched for propensity score (odds ratio 1.9, 95\% CI 1.2-3.1). ${ }^{11}$ Despite statistical tools to minimize selection bias, including risk stratification according to Society of Thoracic Surgeons (STS) and Parsonett models and computer matching by off-pump selection propensity scores, concerns still exist regarding the influence of selection bias, individual surgeon variability, and the potential presence of a learning curve. We therefore undertook this analysis of our total experience with beating-heart surgery from its inception in 1995 through 2000. We asked the following questions:

1. Can off-pump surgery be safely incorporated into a surgical practice and be performed by most surgeons?

2. Is there a significant learning curve to the adoption of beating-heart surgery?

3. Does incorporation of off-pump surgery into a surgical practice lead to better overall outcomes?

4. Are the improved outcomes reported with beatingheart surgery related to the technique or to individual surgeon experience and expertise?

\section{Patients and Methods}

A retrospective review of prospectively collected data entered into an STS computerized database from the Cardiopulmonary Research Science and Technology Institute identified preoperative risk factors, operative variables, and postoperative outcomes from 12,540 patients who underwent isolated CABG from January 1995 through December 2000. This database serves as the outcomes analysis tool for a group averaging approximately 22 cardiac surgeons annually. Because of personnel turnover, outcomes of a total of 32 surgeons were analyzed.

Data were first summarized with descriptive statistics. Continuous variables are presented as mean \pm SD. Categoric and nominal variables are summarized as frequencies and proportions. Traditional univariate and multivariate analyses were carried out to compare risk factors and outcomes between on-pump and offpump surgery groups with the SPSS statistical software package (SPSS Inc, Chicago, Ill). Variables used in the univariate analysis are listed in Appendix 1. Variables were entered into the multi- variate analysis at a $P$ value of .05 . Definitions of these variables are contained in Appendix 2. Stepwise multiple logistic regression analysis was carried out to determine independent predictors of mortality and morbidity for the entire sample and for the on-pump and off-pump groups.

Data were then stratified into six annual cohorts and compared across time with analysis of variance or $\chi^{2}$ test as appropriate. Average predicted risk scores were calculated for the patient population each year with the STS risk algorithm. Overall observed mortality, risk-adjusted mortality, beating-hart CABG mortality, and $\mathrm{CABG}$ with $\mathrm{CPB}$ mortality rates were plotted by year to evaluate trends in the practice with time. Patient demographic characteristics, preoperative risk factors, and postoperative outcomes were compared between the two groups with the Fisher exact test or $\chi^{2}$ test.

To evaluate the influence of surgeon variability on mortality, we analyzed the group of 10 surgeons for which continuous outcomes existed for a 10-year period, the 5 years before significant off-pump experience and 5 years after the introduction of off-pump surgery. The surgeons with the highest adoption rate of beating-heart surgery were compared with those with the lowest adoption rate, and the influence on outcomes was evaluated. Similarly, we also compared the beating-heart surgery adoption rates of the surgeons with the best outcomes with those of the surgeons with the highest mortality in that same group to see whether the introduction of beating-heart surgery had significantly influenced outcomes.

\section{Results}

All 12,540 patients who underwent isolated CABG during the study period from the beginning of 1995 through the end of 2000 were included. One thousand nine hundred fifteen procedures $(15 \%)$ were performed with the heart beating and without $\mathrm{CPB}$. The proportion of $\mathrm{CABG}$ operations performed without $\mathrm{CPB}$ gradually increased, from $1.2 \%$ of all CABG procedures in 1995 to 34\% in 2000 (Figure 1). A single bypass through a limited access incision (MIDCAB) was the predominant procedure in 1995 and 1996, representing $38 \%$ and $41 \%$ of beating-heart cases, whereas a multivessel sternotomy approach comprised most of the procedures in the later years (Figure 2). Adoption of multivessel off-pump approach increased more rapidly in 1998 with the introduction of techniques (Lima sutures) and technology (suction positioners) to more easily access the lateral wall. Table 1 lists the percentages of all grafts that were arterial in both the on-pump and off-pump groups. All MIDCAB procedures (single vessel, limited access) were performed with arterial grafts.

The number of surgeons performing beating-heart surgery started with 2 in 1995 and gradually increased, so that by 2000 all 22 surgeons currently practicing were performing at least some cases off pump. By the last year of the study, 7 of the 22 surgeons (32\%) had performed at least 50 operations off pump, and 5 surgeons $(23 \%)$ had performed at least a 100 CABG procedures off pump. In 2000 offpump surgery represented more than $50 \%$ of all CABG 


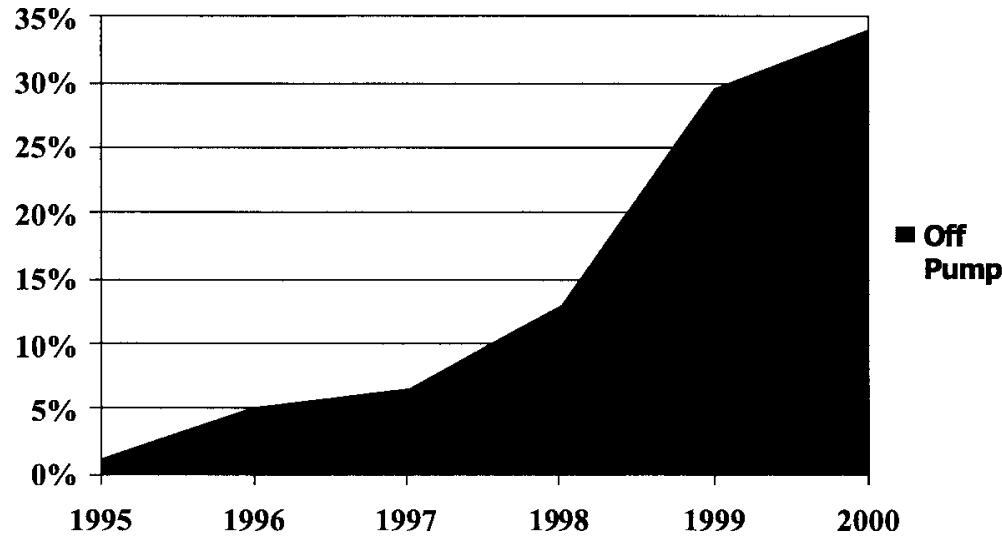

Figure 1. Adoption rate of beating-heart surgery. Shaded area represents percentages of all isolated CABG procedures performed off-pump from 1995 through 2000.

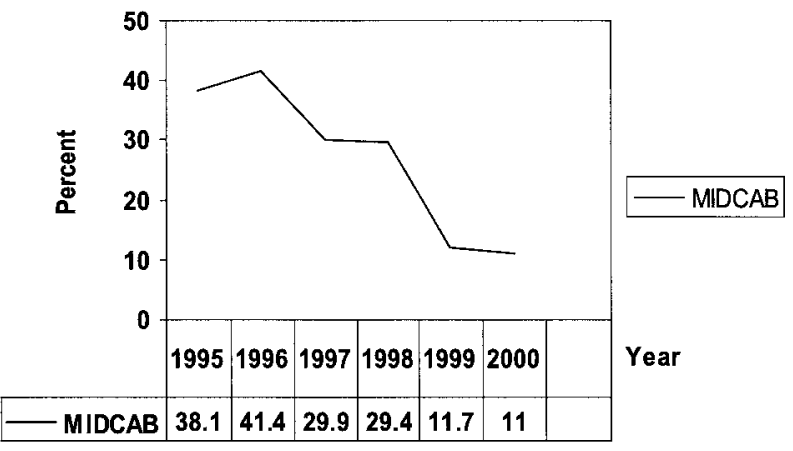

Figure 2. Percentages of beating-heart procedures performed as single-vessel (MIDCAB) rather than multivessel bypass.

cases for 6 surgeons (32\%) and at least $10 \%$ of cases for 11 surgeons $(50 \%)$. However, $73 \%$ of all off pump procedures were performed by 6 surgeons, and 8 surgeons (36\%) were not performing off-pump surgery routinely $(<10$ cases in 2000).

Patient selection was at the individual surgeon's discretion and changed with the year of the procedure and the experience of the surgeon. In the earlier years and with surgeons with little experience, selection tended to toward elective cases requiring a limited number of grafts (2 or 3 ) to the anterior surface of the heart. Patients in unstable condition, those undergoing reoperation, and those requiring multiple bypasses on the lateral surface were generally operated on with CPB during the early years of the experience. In the later years, and as individual surgeon experience increased, minimal selection criteria existed for the experienced surgeons, except that emergency and salvage operations were preferentially preformed with $\mathrm{CPB}$. $\mathrm{Pa}$ tients undergoing beating-heart surgery had a higher predicted risk according to the STS risk algorithm, with a predicted mortality of $3.13 \%$ versus $2.80 \%$ in the conven-
TABLE 1. Types of grafts used

\begin{tabular}{lcc}
\hline & Off-pump group & On-pump group \\
\hline Patients & 1929 & 10,631 \\
Total grafts & 4727 & 36,566 \\
$\quad$ Arterial & $2373(50.2 \%)$ & $10,466(28.6 \%)$ \\
Venous & $2354(49.8 \%)$ & $26,100(71.4 \%)$ \\
Grafts in single-vessel bypass & 573 & 254 \\
Arterial & $471(82.2 \%)$ & $113(44.5 \%)$ \\
Venous & $102(17.8 \%)$ & $141(55.5 \%)$ \\
Grafts in multivessel bypass & 4154 & 36,313 \\
Arterial (\%) & $1902(45.8 \%)$ & $10,354(28.5 \%)$ \\
Venous (\%) & $2252(54.2 \%)$ & $25,959(71.5 \%)$ \\
Mean grafts per case & 2.73 & 2.85 \\
\hline
\end{tabular}

tional CABG group $(P<.004)$. Analysis of 23 preoperative risk variables showed that patients in the off-pump group were more likely to be female and to have chronic lung disease, renal failure, and previous bypass surgery, whereas those in the on-pump group were more likely to have a history of smoking, a family history of coronary artery disease, hypercholesterolemia, previous myocardial infarction, cardiogenic shock, a need for cardiopulmonary resuscitation on the way to the operating room, and three-vessel and left main coronary artery disease (Table 2).

The mean number of grafts performed in the off-pump group was less than that in the CPB group (2.41 vs 2.8); however, there were a larger number of single-vessel bypass operations (MIDCAB procedures) in the beating-heart group than the on-pump group $(573,30 \%$, vs $265,2.5 \%)$. When these single-vessel bypasses were eliminated from the comparison, no significant difference existed between groups, with an average of 2.73 bypasses performed in the off-pump cohort versus 2.88 in the CPB group. There was a conversion rate of $2.9 \%$ from off-pump to on-pump surgery because of either inability to revascularize intended vessels or hemodynamic instability. Because of limitations of the 
TABLE 2. Risk factors with higher frequencies in the off-pump and on-pump groups

\begin{tabular}{|c|c|c|c|c|}
\hline Variable & $\begin{array}{c}\text { Off-pump } \\
\text { group (\%) }\end{array}$ & $\begin{array}{c}\text { On-pump } \\
\text { group (\%) }\end{array}$ & $\chi^{2}$ & $P$ value \\
\hline Female gender & 33.4 & 24.3 & 69.92 & $<.001$ \\
\hline History of smoking & 57.1 & 61.1 & 10.59 & .001 \\
\hline Diabetes & 30.6 & 28.7 & 2.29 & .095 \\
\hline History of coronary artery disease & 46.1 & 54.2 & 42.27 & $<.001$ \\
\hline Hypercholesterolemia & 50.3 & 55.1 & 14.9 & .001 \\
\hline Renal failure & 4.8 & 3.0 & 15.69 & $<.001$ \\
\hline History of cerebrovascular accident & 5.5 & 5.6 & & NS \\
\hline Chronic lung disease & 5.3 & 2.6 & 38.95 & $<.001$ \\
\hline Peripheral vascular disease & 12.5 & 13.4 & & NS \\
\hline Cerebrovascular disease & 10.3 & 10.3 & & NS \\
\hline Reoperative procedure & 14.8 & 7.7 & 79.35 & $<.001$ \\
\hline Previous myocardial infarction & 40.7 & 51.9 & 80.788 & $<.001$ \\
\hline Congestive heart failure & 12.8 & 13.0 & & NS \\
\hline Cardiogenic shock & 0.7 & 1.7 & 9.77 & .002 \\
\hline Cardiopulmonary resuscitation & 0.8 & 1.7 & 9.25 & .002 \\
\hline Atrial fibrillation & 5.4 & 5.2 & & NS \\
\hline Canadian Cardiovascular Society functional class IV & 68.0 & 72.2 & & NS \\
\hline New York Heart Association functional class III & 27.3 & 20.0 & & NS \\
\hline New York Heart Association functional class IV & 67.3 & 72.0 & & NS \\
\hline Preoperative inotropic support & 1.8 & 2.1 & & NS \\
\hline $1-2$ diseased vessels & 18.3 & 2.8 & & $<.001$ \\
\hline 3 or more diseased vessels & 59.6 & 826 & & $<.001$ \\
\hline Left main disease $>50 \%$ & 17.3 & 23.8 & 38.29 & $<.001$ \\
\hline
\end{tabular}

NS, Not significant.

database, conversion data were not captured until the year 2000 , so these patients were analyzed with the on-pump group.

The overall mortality was $3.22 \%$. Observed mortality in the off-pump group was $1.9 \%$, compared with $3.5 \%$ in the on-pump group $(P<.001)$. Risk-adjusted mortality was $1.88 \%$ in the off-pump group and $3.87 \%$ in the on-pump group $(P<.001)$. Observed mortality, predicted risk, and risk adjusted mortality by year are shown in Figures 3 through 5. Multiple logistic regression analysis of the total population revealed that the use of $\mathrm{CPB}$ was an independent risk factor for mortality (odds ratio 2.198 , 95\% CI 1.373.52). Other risk factors predictive of mortality among all patients are listed in Table 3. Separate analyses of on-pump and off-pump groups revealed only age, female gender, and renal failure to be predictive of mortality in the off-pump group, whereas age, female gender, renal failure, peripheral vascular disease, cerebrovascular disease, reoperation, congestive heart failure, cardiogenic shock, left main coronary artery disease, ejection fraction, salvage operation, and emergency operation were all predictive risk factors in the on-pump group (Table 4).

We analyzed our results by year in an attempt to address the influences of patient selection and surgeon experience. As shown in Figures 3 through 5, both observed and riskadjusted mortalities were consistently higher in the CABG with CPB group. We also analyzed the off-pump results by year and surgeon experience in an attempt to discern whether the individual surgeon, surgeon experience, or expertise was a significant variable in outcomes. Figure 6 illustrates the annual mortality for a composite of the 3 surgeons with the highest adoption rate of beating-heart surgery compared with the 3 lowest adopters in the 5 years before and after adoption. There did appear to be a divergence in the curves in 1999 and 2000. Figure 7 illustrates the adoption rates of beating-heart surgery for the groups of surgeons with the highest and the lowest mortalities in the 5 years before the initiation of beating-heart surgery. The 5 -year composite mean operative mortality of the 3 surgeons who were the highest adopters was $2.9 \%$, whereas that of the 3 lowest adopters was $4.0 \%(P<.001)$.

We also examined the influence of the introduction of beating-heart surgery on morbidity and complications for beating-heart surgery compared with conventional CABG (Table 5). In aggregate, beating-heart surgery had significantly lower rates of transfusion, return to the operating room for bleeding, need for prolonged ventilation, and atrial fibrillation, and it was also associated with a shorter hospital stay. The incidence of neurologic events was lower in the off-pump group ( $1.5 \%$ vs $2.2 \%$ ) as was the rate of perioperative myocardial infarction $(0.78 \%$ vs $1.8 \%)$, but neither difference was statistically significant. These results were also examined for trends, and in all years there was a significantly lower incidence of morbidity in the off-pump 


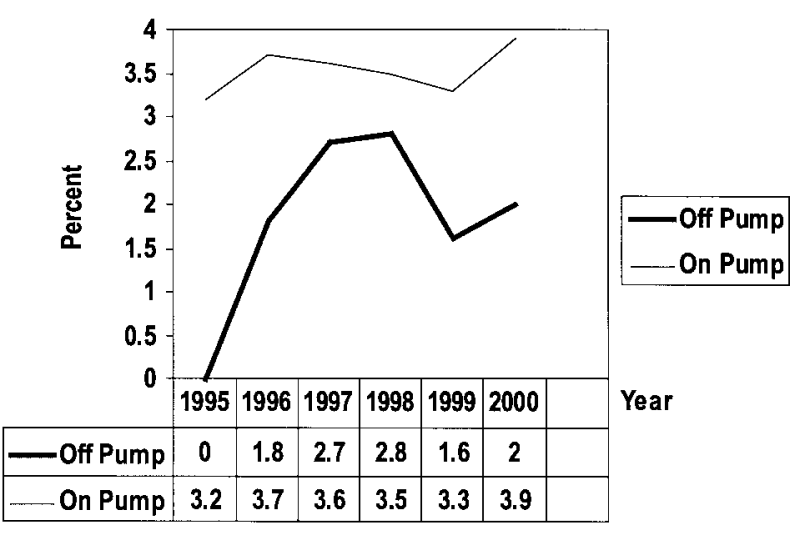

Figure 3. Trends in observed mortality for off-pump (heavy rule) and on-pump (light rule) groups from 1995 through 2000.

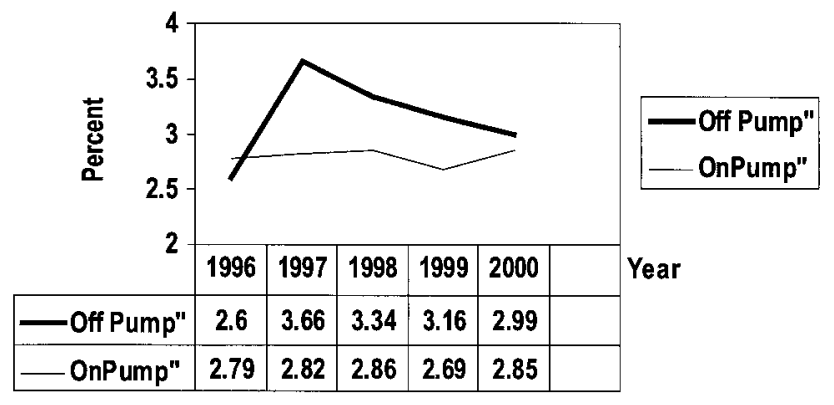

Figure 4. Trends in predicted risk according to STS risk algorithm for off-pump (heavy rule) and on-pump (light rule) groups from 1996 through 2000. Series $P=.004 ; 1999 P=.023 ; P$ not significant in other years.

group. Other postoperative complications, including renal failure, showed no significant difference between beatingheart surgery and conventional $\mathrm{CABG}$.

\section{Discussion}

We undertook this analysis in an attempt to learn whether the gradual integration of beating-heart techniques could lead to better overall outcomes. Additionally, the roles in improved off-pump outcomes of selection bias and individual variability in experience and expertise of the surgeon were investigated. We also examined trends in adoption rates and looked for a detectable learning curve with beating-heart surgery to determine any effects on outcome.

We began our beating-heart experience in 1995 with the MIDCAB procedure. This experience peaked in 1998, with 85 procedures representing $29 \%$ of our beating-heart surgery, but by 2000 MIDCAB represented only $11 \%$ of our beating-heart procedures and $3.7 \%$ of our total CABG volume. The success of catheter-based intervention, the gatekeeper effect of cardiology referrals, and the technical challenges of MIDCAB have now relegated this procedure to a

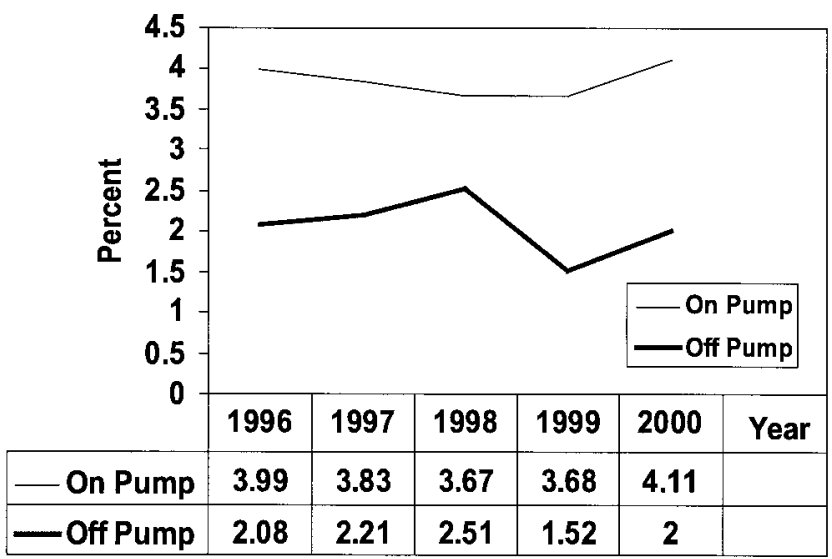

Figure 5. Trends in risk adjusted mortality for off-pump (heavy rule) and on-pump (light rule) groups from 1996 through 2000. $P<$ .01 for all years.

TABLE 3. Multiple logistic regression analysis of 23 preoperative variables with respect to mortality outcome

\begin{tabular}{lccc}
\hline Risk factor & $\begin{array}{l}\text { Odds } \\
\text { ratio }\end{array}$ & $\mathbf{9 5 \%} \mathbf{C l}$ & $\boldsymbol{P}$ value \\
\hline Cardiopulmonary resuscitation & 2.737 & $1.274-5.881$ & .01 \\
Reoperation & 2.616 & $1.803-3.794$ & $<.001$ \\
Cardiogenic shock & 2.327 & $0.93-5.567$ & .05 \\
CPB & 2.198 & $1.37-3.52$ & .001 \\
Female gender & 1.635 & $1.214-2.202$ & .001 \\
Left main coronary artery disease & 1.349 & $1.001-1.817$ & .049 \\
Low ejection fraction & 1.014 & $1.002-1.026$ & .02 \\
Age & 0.953 & $0.938-0.968$ & $<.001$ \\
\hline
\end{tabular}

minor position. As techniques and technology improved, the clinical benefit became more apparent, and the ability to apply off-pump techniques to most multivessel surgical cases increased, our adoption rate of multivessel beatingheart surgery by sternotomy has increased.

The initial experience with beating-heart surgery in our surgical group practice was limited only to a few surgeons. The procedure gradually became more integrated. All surgeons had performed at least some beating-heart procedures by 2000 . However, most off-pump procedures (73\% in the year 2000) are still performed by the 6 surgeons who first and most avidly adopted beating-heart techniques. Indeed, some surgeons still seldom use beating-heart techniques. Although in 2000 all surgeons performed some beatingheart surgery, 8 surgeons performed fewer than 10 cases during the year.

There clearly has been selection bias in choosing patients for beating-heart surgery. In the overall cohort, as well as in each annual cohort after 1996, patients at higher risk were preferentially chosen for beating-heart operations. Patients preferentially selected for off-pump procedures included those traditionally considered at high-risk for $\mathrm{CABG}$, in- 


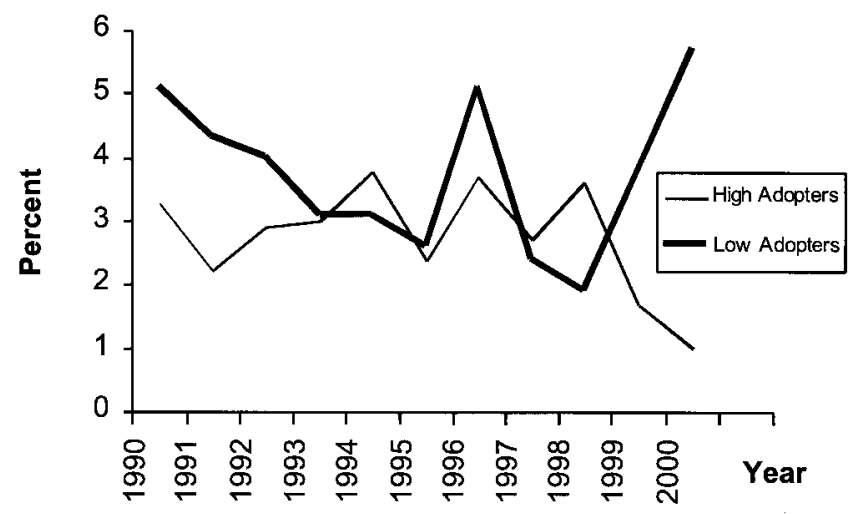

Figure 6. CABG-associated mortalities among patients of high adopters (light rule) and low adopters (heavy rule) of beatingheart surgery from 1990 through 2000.

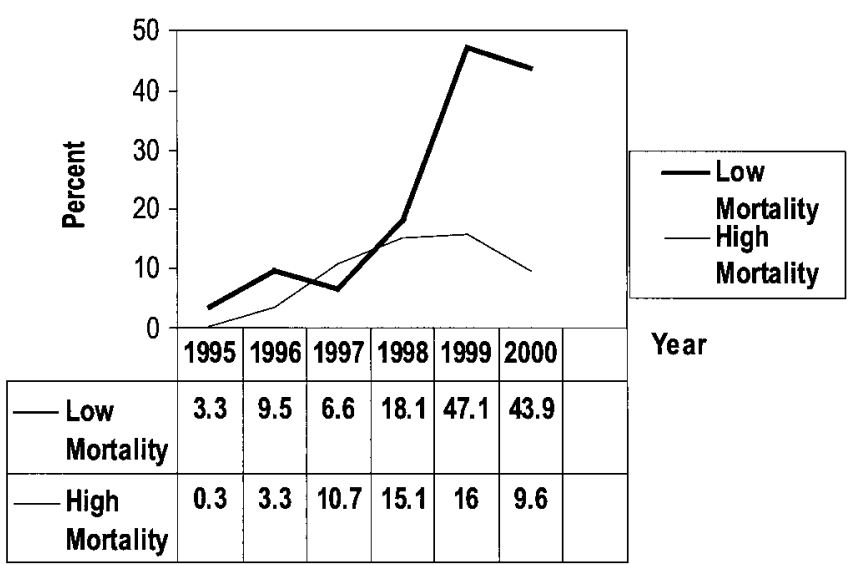

Figure 7. Adoption rates of beating-heart surgery among 3 surgeons with highest mortality (light rule) and 3 surgeons with lowest mortality (heavy rule) from 1995 through 2000.

TABLE 4. Risk factors predictive of mortality in on-pump and off-pump CABG groups

\begin{tabular}{|c|c|c|c|c|}
\hline \multirow[b]{2}{*}{ Risk factor } & \multicolumn{2}{|c|}{ On-pump group } & \multicolumn{2}{|c|}{ Off-pump group } \\
\hline & Odds ratio & $95 \% \mathrm{Cl}$ & Odds ratio & $95 \% \mathrm{Cl}$ \\
\hline Age & 1.055 & $1.042-1.067$ & 1.062 & $1.021-1.105$ \\
\hline Female gender & 0.533 & $0.419-0.677$ & 0.408 & $0.183-0.909$ \\
\hline Renal failure & 2.03 & $1.326-3.109$ & 2.371 & $0.710-7.914$ \\
\hline Peripheral vascular disease & 1.845 & $1.419-2.398$ & & \\
\hline Cerebrovascular disease & 1.167 & $0.582-1.698$ & & \\
\hline Reoperation & 2.849 & $2.090-3.883$ & & \\
\hline Congestive heart failure & 1.426 & $1.074-1.892$ & & \\
\hline Cardiogenic shock & 2.807 & $1.649-4.778$ & & \\
\hline Left main coronary artery disease $>50 \%$ & 1.323 & $1.034-1.693$ & & \\
\hline Ejection fraction & 0.982 & $0.972-0.991$ & & \\
\hline Salvage operation & 6.607 & $3.515-12.420$ & & \\
\hline Emergency operation & 1.682 & $1.085-2.606$ & & \\
\hline
\end{tabular}

cluding women, patients undergoing reoperation, and those with preexisting renal failure, although the on-pump surgery group had more three-vessel and left main coronary artery disease. We fully acknowledge that the standard risk algorithms for $\mathrm{CABG}$ mortality were developed for $\mathrm{CABG}$ with $\mathrm{CPB}$ and many not accurately reflect risk factors in beatingheart cases.

On an annual basis, both observed and risk-adjusted mortalities were consistently lower in the off-pump cohort. On the basis of the overall observed mortality in 1998 through 2000, it appears that the adoption of beating-heart techniques has led to a trend in lower mortality in the overall CABG population (Figure 8). The mean observed operative mortality for all isolated CABG procedures decreased to $3.2 \%$ in the period 1995 through $2000(\mathrm{n}=12,540)$ from $4.0 \%$ in the period 1990 through $1994(\mathrm{n}=6468$, $P=$.0482).

An unanswered question has been whether the improved outcomes observed with beating-heart surgery stem from
TABLE 5. Postoperative complications and hospital stays for on-pump and off-pump cohorts

\begin{tabular}{lccr}
\hline & $\begin{array}{c}\text { Off-pump } \\
\text { group }\end{array}$ & $\begin{array}{c}\text { On-pump } \\
\text { group }\end{array}$ & $\boldsymbol{P}$ value \\
\hline Transfusion (\%) & 27.6 & 53.8 & $<.001$ \\
Reoperative bleeding (\%) & 1.9 & 3.4 & $<.001$ \\
Prolonged ventilation (\%) & 4.6 & 10.5 & $<.001$ \\
Renal failure (\%) & 2.6 & 3.9 & $<.001$ \\
Atrial fibrillation (\%) & 16.4 & 22.5 & $<.001$ \\
Neurologic complications (\%) & 1.5 & 2.2 & .251 \\
Hospital stay (d) & 5.95 & 7.33 & $<.001$ \\
\hline
\end{tabular}

more experienced surgeons with better established outcomes preferentially adopting beating-heart techniques rather than from the intrinsic merits of beating-heart surgery. Mortality outcomes from 1990 through 1994 demonstrate that the surgeons with better outcomes did have a higher adoption rate of off-pump techniques than did the 


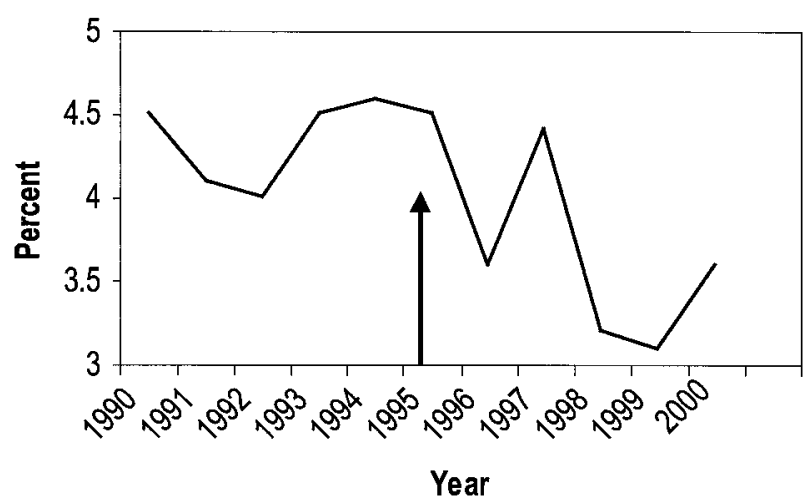

Figure 8. Annual trend in observed operative mortality from 1990 through 2000. Arrow indicates introduction of beating-heart surgery.

group with higher mortality. This indicates that the outcome improvement observed with off-pump surgery was not due solely to the technique and that the individual surgeon may have played a significant role. Stated differently, the use of beating-heart techniques may not lead to improved outcomes for all surgeons. However, in examining trends of mortality outcomes among surgeons who had the earliest high adoption rate of off-pump surgery (high adopters) and those of low adopters, a mortality benefit trend can be seen even among the high adopters with the best overall outcomes in the last 2 years of the study.

In conclusion, and in response to the questions posed in the introduction, off-pump surgery can be safely incorporated into a surgical group practice and can be performed by most surgeons. However, adoption rate varies by individual, and it is not clear that adoption by all surgeons leads to improved outcomes. There remains a core group of surgeons who perform most of the off-pump operations. There does not appear to be a significant learning curve, as evidenced in the annual cohort analysis, which shows a consistently lower mortality in the off-pump group despite a higher predicted risk. Although there were consistently better outcomes with beating-heart surgery, the individual surgeon was a variable that probably contributed along with off-pump techniques themselves to the better surgical outcomes. Surgeons with a lower mortality in the 5-year period before the beating-heart program tended to adopt beatingheart techniques earlier and to a greater degree. Also, mortality outcomes appeared to improve for the high adopters of beating-heart surgery but not for the low adopters. Finally, gradual adoption and integration of beating-heart surgery, as reflected in the last 2 years of the study, led to a trend toward overall improved CABG outcomes.

\section{References}

1. Mack MJ. Pro: beating heart surgery for coronary revascularization: is it the most important development since the introduction of the heartlung machine? Ann Thorac Surg. 2000;70:1774-8.

2. Ascione R, Lloyd CT, Gomes WJ, Caputo M, Bryan AJ, Angelini GD. Beating versus arrested heart revascularization: evaluation of myocardial function in a prospective randomized study. Eur J Cardiothorac Surg. 1999;15:685-90.

3. Diegeler A, Hirsch R, Schneider F, Schilling LO, Falk V, Rauch T, et al. Neuromonitoring and neurocognitive outcome in off pump versus conventional coronary bypass operation. Ann Thorac Surg. 2000;69: 1162-6.

4. Ricci M, Karamanoukian HL, Abraham R, Von Fricken K, D’Ancona $\mathrm{G}$, Choi S, et al. Stroke in octogenarians undergoing coronary artery surgery with and without cardiopulmonary bypass. Ann Thorac Surg. 2000;69:1471-5.

5. Stamou SC, Dangas G, Dullum MK, Pfister AJ, Boyce SW, Bafi AS, et al. Beating heart surgery in octogenarians: perioperative outcome and comparison with younger age groups. Ann Thorac Surg. 2000;69: 1140-5.

6. Boyd WD, Desai ND, Del Rizzo DF, Novick RJ, McKenzie FN, Menkis AH. Off pump surgery decreases postoperative complications and resource utilization in the elderly. Ann Thorac Surg. 1999;68: 1490-3.

7. Ascione R, Lloyd CT, Underwood MJ, Gomes WJ, Angelini GD. On-pump versus off-pump coronary revascularization: evaluation of renal function. Ann Thorac Surg. 1999;68:493-8.

8. Hart JC, Spooner TH, Pym J, Flavin TF, Edgerton JR, Mack MJ, et al. A review of 1,582 consecutive Octopus off-pump coronary bypass patients. Ann Thorac Surg. 2000;70:1017-20.

9. Arom KV, Flavin TF, Emery RW, Kshettry VR, Petersen RJ, Janey PA. Is low ejection fraction safe for off-pump coronary bypass operation? Ann Thorac Surg. 2000;70:1021-5.

10. Yokoyama T, Baumgartner FJ, Gheissari A, Capouya ER, Panagiotides GP, Declusin RJ. Off-pump versus on-pump coronary bypass in high-risk subgroups. Ann Thorac Surg. 2000;70:1546-50.

11. Trehan N, Mishra YK, Malhotra R, Sharma KK, Mehta Y, Shrivastava S. Off-pump redo coronary artery bypass grafting. Ann Thorac Surg. 2000;70:1026-9.

12. Bergsland J, Hasnain S, Lajos TZ, Salerno TA. Elimination of cardiopulmonary bypass: a prime goal in reoperative coronary artery bypass surgery. Euro J Cardiothorac Surg. 1998;14:59-63.

13. Magee MJ, Jablonski KA, Stamou SC, Pfister AJ, Dewey TM, Dullum $\mathrm{MK}$, et al. Elimination of cardiopulmonary bypass improves early survival for multivessel coronary artery bypass patients. Ann Thorac Surg. 2002;73:1196-202.

\section{Appendix 1}

\section{Variables Used in Univariate Analysis}

The following variables were used in the univariate analysis and entered into multivariate analysis at $P \leq .05$.

- Gender

- Current smoking

- History of coronary artery disease

- Diabetes

- Hypercholesterolemia

- Renal failure

- Hypertension

- Cerebrovascular accident

- Chronic lung disease including chronic obstructive pulmonary disease

- Peripheral vascular disease

- Cerebrovascular disease

- Reoperative status

- Myocardial infarction

- Congestive heart failure 
- Angina

- Cardiogenic

- Resuscitation

- Canadian Cardiovascular Society functional class

- New York Heart Association functional class

- Inotrope use

- Left main coronary artery disease

- Status

- Predicted risk

- Number of vessels

- Pump use

- Ejection fraction

- Age

\section{Appendix 2}

\section{Definition of Data Elements}

Gender. Male or female

Current smoking. Patients having smoked cigarettes within 1 month of surgery

History of coronary artery disease. Whether any direct relatives (parents, siblings, children) have had any of the following at younger than 55 years:

1. Angina

2. Myocardial infarction

3. Sudden cardiac death without obvious cause

Diabetes. A history of diabetes, regardless of duration or need for antidiabetic agents

Hypercholesterolemia. History of hypercholesterolemia diagnosed or treated by a physician with documentation of any of the following:

1. Total cholesterol greater than $200 \mathrm{mg} / \mathrm{dL}$

2. Low-density lipoproteins greater than $130 \mathrm{mg} / \mathrm{dL}$

3. High-density lipoproteins less than $30 \mathrm{mg} / \mathrm{dL}$

4. Admission cholesterol greater than $200 \mathrm{mg} / \mathrm{dL}$

Renal failure. Creatinine level greater than $2.0 \mathrm{mg} / \mathrm{dL}$

Hypertension. Any of the following:

1. Documented history of hypertension diagnosed and treated with medication, diet, or exercise

2. Systolic blood pressure greater than $140 \mathrm{~mm} \mathrm{Hg}$ or diastolic blood pressure greater than $90 \mathrm{~mm} \mathrm{Hg}$ on at least two occasions

Cerebrovascular accident. Central neurologic deficit lasting longer than 24 hours

Chronic lung disease. Presence of chronic lung disease, including chronic obstructive pulmonary disease

Peripheral vascular disease. Peripheral vascular disease as indicated by any of the following:

1. Claudication either with exertion or rest

2. Amputation for arterial insufficiency

3. Aortoiliac occlusive disease reconstruction

4. Peripheral vascular bypass surgery, angioplasty, or stent

5. Documented abdominal aortic aneurysm (AAA), AAA repair, or stent

6. Documented positive results of noninvasive testing

Cerebrovascular disease. Cerebrovascular disease as documented by any of the following:

1. Unresponsive coma longer than 24 hours

2. Cerebrovascular accident (symptoms $>72$ hours after onset)

3. Reversible ischemic neurologic deficit (recovery within 72 hours)
4. Transient ischemic attack (recovery within 24 hours)

5. Noninvasive carotid test with $75 \%$ occlusion

Reoperative status. Previous CABG by any approach

Myocardial infarction. Patient hospitalized for a myocardial infarction documented by two of the following four criteria:

1. Prolonged ( $>20$ minutes) typical chest pain not relieved by rest and/or nitrates

2. Enzyme level elevation, either (1) creatine kinase isoenzyme MB representing more than $5 \%$ of total creatine kinase, (2) creatine kinase more than twice normal, (3) lactate dehydrogenase subtype 1 greater than lactate dehydrogenase subtype 2, or (4) troponin greater than $0.2 \mu \mathrm{g} / \mathrm{mL}$

3. New wall motion abnormalities

4. Serial electrocardiograms ( $\geq 2$ ) showing changes from baseline or serially in ST-T or Q waves 0.03 second in width or at least a third of the total QRS complex in two or more contiguous leads

Congestive heart failure. Physician diagnosis of congestive heart failure within 2 weeks before procedure as documented by any of the following:

1. Paroxysmal nocturnal dyspnea

2. Dyspnea on exertion related to heart failure

3. Chest radiograph showing pulmonary congestion and treatment with diuretic or digoxin

Angina. Angina pectoris

Cardiogenic shock. Clinical state of hypoperfusion at the time of the procedure according to either of the following criteria:

1. Systolic blood pressure less than $80 \mathrm{~mm} \mathrm{Hg}$ or cardiac index less than 1.8 despite maximal treatment

2. Intravenous inotropic support or intra-arterial balloon pump necessary to maintain systolic blood pressure greater than 80 $\mathrm{mm} \mathrm{Hg}$ or CI of more than 1.8

Resuscitation. Need for cardiopulmonary resuscitation within 1 hour of the start of the procedure

Canadian Cardiovascular Society functional class. Highest functional class leading to episode of hospitalization or intervention according to following scale:

- 0 , No angina

- I, Ordinary physical activity, such as walking or climbing the stairs does not cause angina. Angina may occur with strenuous, rapid, or prolonged exertion at work or recreation.

- II, There is slight limitation of ordinary activity. Angina may occur with moderate activity, such as walking or climbing stairs rapidly, walking uphill, walking or stair climbing after meals or in the cold, or walking more than 2 blocks.

- III, There is marked limitation of ordinary physical activity. Angina may occur after walking 1 or 2 blocks or climbing 1 flight of stairs under normal conditions at a normal pace.

- IV, There is an inability to carry on any physical activity without discomfort; angina may be present at rest.

New York Heart Association functional class. Highest functional class leading to episode of hospitalization or intervention according to following scale:

- I, Patients with cardiac disease but without resulting limitation of physical activity. Ordinary physical activity does not cause undue fatigue, palpitations, dyspnea, or anginal pain.

- II, Patients with cardiac disease resulting in slight limitation of physical activity. They are comfortable at rest. Less than ordinary physical activity results in fatigue, palpitations, dyspnea, or anginal pain.

- III, Patients with cardiac disease resulting in marked limitation of physical activity. They are comfortable at rest. Less 
than ordinary physical activity results in fatigue, palpitations, dyspnea, or anginal pain.

- IV, Patients with cardiac disease resulting in inability to carry on any physical activity without discomfort. Symptoms of cardiac insufficiency or of the anginal syndrome may be present even at rest. If any physical activity is undertaken, discomfort is increased.

Inotropic support. Preoperative use of inotropes

Left main coronary artery disease. Left main coronary artery disease as indicated by greater than $50 \%$ compromise of vessel diameter in any angiographic view

Elective status. Procedure could be deferred without increased risk of compromised cardiac outcome.

Urgent status. Operation meeting all the following conditions:

1. Not elective

2. Not emergency

3. Required during the same hospitalization to minimize chance of further clinical deterioration

4. Worsening, sudden chest pain, congestive heart failure, acute myocardial infarction, compelling coronary anatomy, intra-aortic balloon pump, unstable angina with intravenous nitroglycerin or rest angina may be included

Emergency status. Clinical status including any of the following:

1. Ischemic dysfunction (any of the following): (1) ongoing ischemia including rest angina despite maximal medical therapy (medical or intra-aortic balloon pump), (2) acute evolving myocardial infarction within 24 hours before surgery, (3) pulmonary edema requiring intubation

2. Mechanical dysfunction (either of the following): (1) shock with circulatory support, (2) shock without circulatory support

Predicted risk. A number calculated from STS algorithm

Number of vessels. Number of vessels bypassed

Pump use. Use of CPB, with off-pump equal to crossclamp and perfusion time of zero and on-pump equal to crossclamp or perfusion time of at least 1 minute

Ejection fraction. Percentage of the blood emptied from the ventricle at the end of the contraction according to the most recent determination before the intervention

Age. Age determined from the date of birth to the date of surgery

\section{Discussion}

Dr Antonio M. Calafiore (Chieti, Italy). I congratulate Mack and coworkers for this detailed demonstration of the possibility of introducing myocardial revascularization without CPB in a large clinical practice with excellent clinical results.

Retrospective studies are important because they represent our daily life, where the choice of the surgical strategy depends on the patient, on the patient's clinical characteristics, and on the patient's specific coronary anatomy. Multiple logistic regression analysis is able to limit biases related to patient selection and gives us an idea about the effectiveness of our choices. This study clearly shows a reduction in the mortality rate of nearly $1 \%$ in the period 1996 through 2000 in comparison with the period 1990 through 1995. This positive aspect is very likely due to the introduction of off-pump surgery.

Dr Mack, in your presentation you did not talk about patients who had their procedures converted from off pump to on pump.
Had you no cases to report? If any, were conversions analyzed in the on-pump or off-pump group?

The increasing number of surgeons performing off-pump surgery demonstrates the increasing technical feasibility of the procedure. Which was the main technical determinant of increasing the percentage of off-pump cases? In particular, what was the role of lateral grafting?

Looking at the data, with the increasing percentage of off-pump cases, the predicted risk of the off-pump group in the year 2000 is similar to the on-pump group's predicted risk. However, the observed and risk-adjusted mortalities in the on-pump group are double those of the off-pump group. Can you comment on this aspect? It seems that part of the benefit to the off-pump group is related not only to intrinsic good results but also to higher mortality in the on-pump group. Furthermore, the mortality in the year 2000 of the low adopters, nearly $6 \%$, seems to me to be too high to be explained simply by refusal to use off-pump surgery.

In your presentation you showed with a multivariate analysis that CPB is an independent risk factor for higher early mortality. Other end points, such as transfusion rate, incidence of atrial fibrillation, and so on, were analyzed only with a univariate analysis. One important end point, cerebrovascular complications, is similar in both groups, but nothing was said about the incidence of acute myocardial infarction, one of the most important end points in coronary artery surgery. Can you tell us if there was any difference between the two groups?

Dr Mack. Dr Calafiore, we appreciate the leading role that you have served in this minimally invasive surgery and indeed have followed closely your example of integrating these approaches into our practice. I will try to answer as many of your questions as I can.

First, patients who had the procedure converted from off pump to on pump were treated as part of the off-pump group because this was an intent-to-treat analysis. The conversion rate for the whole series was $1.6 \%$. The conversion rate for the last year of the study was $0.6 \%$. The broadening in the latter years of the study I think was due to increasing surgeon comfort with the techniques and to advances in technology that allowed not only stabilization but especially post exposure to the posterior aspect of the myocardial coronary arteries. We have tried to take a gradual integrated approach in which a few surgeons started with this and then it gradually permeated out through the practice. I think by 1998 , when the experience significantly increased, it was both a matter of our own surgeons being more comfortable with the techniques and the technology allowing broader application.

Regarding the on-pump mortality being high in the latter years of the study, we agree with your observation. This is a retrospective analysis, with all the inherent problems associated with the methodology. On the other hand, we are a large, diverse group practice that incorporates all variations in cardiac surgery, and I think that we are a microcosm that mirrors what really is going on out there. Like a large mutual fund that cannot vary much from the stock market, every year our mortality mirrors that of the STS.

So we have surgeons with great outcomes and surgeons with not so great outcomes. I think that our analysis shows that the surgeons with good outcomes further improved those outcomes with the adoption beating-heart surgery. I do not think we know whether the adoption of beating-heart techniques by surgeons 
whose outcomes are not as good will be helpful or hurtful, and we have been reticent to address that issue or to encourage some of those surgeons to adopt the techniques. Because of the time constraints of the presentation, I did not present multivariate analysis on a lot of the outcomes. They did mirror the univariate analysis.

Perioperative myocardial infarction was the same both onpump and off-pump groups. There was no difference between the two groups. The percentages of perioperative myocardial infarction were $0.78 \%$ in the off-pump group and $1.8 \%$ in the on-pump patients.

Dr Michael C. Maxwell (Charlotte, NC). The people who do not like off-pump surgery commonly claim that the problem is not the pump but the partial clamp placed for sewing proximals. I imagine you have included some MIDCABs or cases without any partial occlusion clamp. Did you analyze those two subgroups? If so, did you find a difference?
Dr Mack. That is a valid point. Yes, we did analyze the groups, eliminating the MIDCAB cases from the analysis and eliminating single-vessel procedures from both groups, which included eliminating the MIDCABs. The outcomes all stayed the same, because the MIDCABs still represented a relatively small proportion of the whole group.

Relevant to the point is that the more you use a partial occlusion clamp on the aorta, the more you negate any positive benefits of eliminating CPB. I think that there have been reports in the medical literature in the last few months of aortic dissection occurring with beating-heart surgery with occlusion of the partial aortic clamp, and I think that the beating heart with higher pressure sometimes against a partial occlusion clamp carries the potential for adverse outcomes. I think that the use of some of the clampless proximal anastomotic techniques that are soon to be introduced may further help address this issue of clamping of the aorta. 